\title{
Atomic-scale structure of $\mathrm{Ga}_{1-x} \operatorname{In}_{x} \mathrm{P}$ alloys measured with extended $\mathrm{x}$-ray absorption fine structure spectroscopy
}

\author{
C. S. Schnohr, L. L. Araujo, P. Kluth, and D. J. Sprouster \\ Department of Electronic Materials Engineering, Research School of Physical Sciences and Engineering, \\ Australian National University, Canberra ACT 0200, Australia \\ G. J. Foran \\ Australian Nuclear Science and Technology Organisation, Menai NSW 2234, Australia \\ M. C. Ridgway \\ Department of Electronic Materials Engineering, Research School of Physical Sciences and Engineering, \\ Australian National University, Canberra ACT 0200, Australia \\ (Received 19 February 2008; revised manuscript received 13 May 2008; published 10 September 2008)
}

\begin{abstract}
Extended x-ray absorption fine structure spectroscopy was used to measure the interatomic distance distributions of the first three nearest-neighbor (NN) shells around Ga and In atoms in $\mathrm{Ga}_{1-x} \mathrm{In}_{x} \mathrm{P}$. The first NN shell has a composition-dependent bimodal distance distribution with a relaxation parameter of $\epsilon=0.80 \pm 0.04$ similar to other III-V ternary alloys. The second $\mathrm{NN}$ shell distance distribution remains multimodal, corresponding to the three different cation-cation pairs but is closer to the virtual-crystal approximation (VCA). The third NN shell mean distance is well approximated by the VCA although the distribution is significantly broadened. Predictive model calculations are discussed in detail where good agreement with experimental results is found. Like in $\mathrm{Ga}_{1-x} \mathrm{In}_{x} \mathrm{As}$, lattice mismatch is accommodated in $\mathrm{Ga}_{1-x} \mathrm{In}_{x} \mathrm{P}$ by both bond-length and bond-angle relaxations although primarily via the latter.
\end{abstract}

DOI: 10.1103/PhysRevB.78.115201

PACS number(s): 61.05.cj, 61.66.Dk, 61.72.uj

\section{INTRODUCTION}

Ternary alloys of the form $\mathrm{A}_{1-x} \mathrm{~B}_{x} \mathrm{C}$ have long been studied due to the potential of tuning their properties with composition $x$ between those of the two end-point binary compounds. The dilute limit is also of interest since many device applications rely on the properties of impurity atoms. From a structural point of view, one important question is how the two different binary lattice constants are accommodated in the atomic-scale structure of the ternary alloy. Many materials, among them $\mathrm{Ga}_{1-x} \operatorname{In}_{x} \mathrm{As}$ and $\mathrm{Ga}_{1-x} \operatorname{In}_{x} \mathrm{P},{ }^{1}$ exhibit a lattice constant that varies linearly with composition $x$ in accordance with Vegard's law. ${ }^{2}$ The so-called virtual-crystal approximation $^{3}$ (VCA) assumes all atoms occupy ideal lattice sites with the lattice constant determined by $x$. All pairs of neighboring atoms have the same bond lengths and the bond angles remain unchanged. In contrast, Pauling and Huggins ${ }^{4}$ assume the conservation of atomic radii, which results in different bond lengths for the A-C and B-C pairs independent of $x$ and identical to those of the binary compounds. The lattice mismatch is accommodated by relaxation in the bond angles from the tetragonal norm. Experiments on a variety of III-V, II-VI, and I-VII ternary alloys have used extended x-ray absorption fine structure (EXAFS) spectroscopy to study the local atomic environment around the absorbing atom. ${ }^{5-10}$ In all cases the first nearest-neighbor (NN) distance distribution was shown to be bimodal and closer to the Pauling limit than the VCA. A number of models have been proposed for ternary structures predicting the first $\mathrm{NN}$ distances. ${ }^{11-14}$ All these calculations yield similar results despite very different assumptions. To achieve a more comprehensive picture, it is therefore necessary to consider higher atomic shells, both theoretically and experimentally. Extensive calculations by Silverman et al. ${ }^{15}$ for $\mathrm{Ga}_{1-x} \mathrm{In}_{x} \mathrm{P}$ alloys predict the interatomic distance distributions of the first and second NN shells as well as the bond angle distributions. Experimentally, similarities to other III-V compounds have been observed for the first $\mathrm{NN}$ shell of $\mathrm{Ga}_{1-x} \mathrm{In}_{x} \mathrm{P}$ in the sole brief report ${ }^{6}$ on this system of which we are aware. There is no experimental data available on the higher shells despite the technological importance of $\mathrm{Ga}_{1-x} \mathrm{In}_{x} \mathrm{P}$ alloys. The direct and relatively high band gap and the low density of deep level traps make this system particularly appropriate for applications such as high electron mobility transistors, field effect transistors in high-speed circuits, power amplifiers, and lasers (Refs. 16 and 17 and references therein). $\mathrm{Ga}_{1-x} \mathrm{In}_{x} \mathrm{P}$ is also a very promising material for solar cells both in spatial and terrestrial applications ${ }^{16,18,19}$ due to high efficiencies and superior radiation resistance; furthermore, recent works on quantum structures demonstrate the continued interest in $\mathrm{Ga}_{1-x} \mathrm{In}_{x} \mathrm{P}$ based systems. ${ }^{16,20}$ To contribute to a more comprehensive understanding of these alloys and to assess the validity of a variety of structural models, we now present a detailed study of the distance distributions of the first three $\mathrm{NN}$ shells around $\mathrm{Ga}$ and $\mathrm{In}$ atoms in $\mathrm{Ga}_{1-x} \mathrm{In}_{x} \mathrm{P}$ (as measured by EXAFS) including the evaluation of both bond lengths and bond angles. Our results are compared to predictive calculations and clearly demonstrate the competing effects of bond-length and bond-angle relaxations.

\section{EXPERIMENTAL DETAILS}

$\mathrm{Ga}_{1-x} \mathrm{In}_{x} \mathrm{P} / \mathrm{AlAs} / \mathrm{GaAs}$ heterostructures were fabricated by metal organic chemical vapor deposition. GaAs substrates 

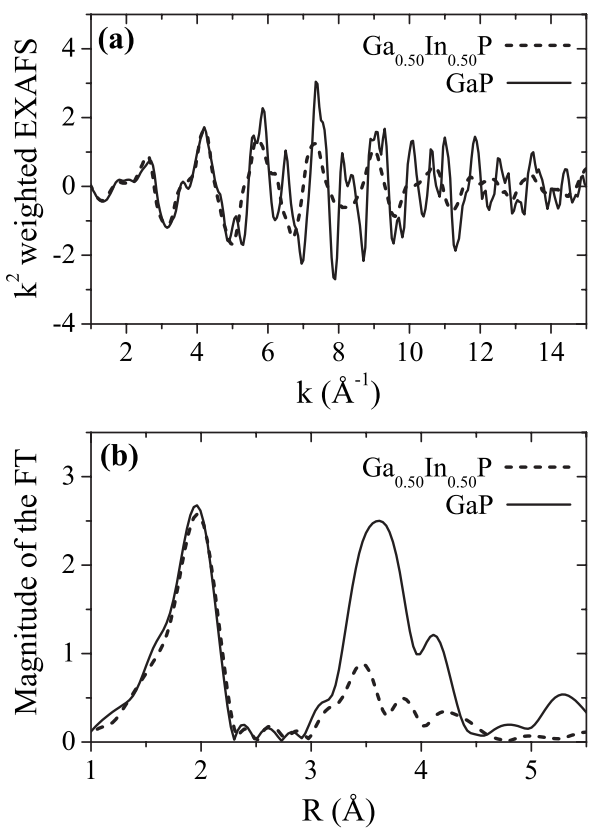

FIG. 1. (a) $k^{2}$-weighted EXAFS spectra of GaP (solid line) and $\mathrm{Ga}_{0.50} \operatorname{In}_{0.50} \mathrm{P}$ (dashed line) recorded at the $\mathrm{Ga} K$ edge versus the photoelectron wave number $k$. (b) Corresponding Fourier transforms as a function of the nonphase-corrected radial distance $R$ from the absorber.

with a miscut of $10^{\circ}$ relative to the (100) direction were used to inhibit ordering of the mixed $\mathrm{Ga} / \mathrm{In}$ sublattice. Compositions $x=0.34 \pm 0.02, x=0.50 \pm 0.02$, and $x=0.70 \pm 0.03$ with a layer thickness of $1.0,2.5$, and $0.5 \mu \mathrm{m}$, respectively, were grown. The thickness of the intermediate AlAs layer was approximately $50 \mathrm{~nm}$. The composition was determined by $\mathrm{x}$-ray diffraction and Rutherford backscattering spectrometry (RBS). RBS measurements in channeling configuration also confirmed the crystallinity of the epitaxial layers.

To prepare samples suitable for EXAFS measurements, the $\mathrm{Ga}_{1-x} \mathrm{In}_{x} \mathrm{P}$ layer was removed from the substrate by selective chemical etching. The samples were first masked with wax to physically support the ternary layer, then the AlAs layer was selectively dissolved in $10 \% \mathrm{HF}$ over $24 \mathrm{~h}$. The wax was then removed from the isolated $\mathrm{Ga}_{1-x} \mathrm{In}_{x} \mathrm{P}$ layer and the samples were finely crushed and mixed with BN. Diluted powder samples of $\mathrm{GaP}$ and $\mathrm{InP}$ were prepared as references. EXAFS measurements at the Ga and In $K$ edges were performed in transmission mode at beam lines 20B and NW10A, respectively, at the Photon Factory (Japan). The measurement temperature was $10-20 \mathrm{~K}$.

The data were processed and analyzed using the IFEFFIT code $^{21}$ and the corresponding user interfaces ATHENA and ARTEMIS. ${ }^{22}$ After background removal, the data were Fourier transformed (FT) over a photoelectron wave-number $(k)$ range of $k=2-14 \AA^{-1}$ for both edges. Figure 1(a) shows the $k^{2}$-weighted EXAFS signal as a function of $k$ measured at the Ga $K$ edge for $\mathrm{GaP}$ and $\mathrm{Ga}_{050} \mathrm{In}_{0.50} \mathrm{P}$. The corresponding FTs are plotted in Fig. 1(b). Figures 2(a) and 2(b) show the data for $\mathrm{Ga}_{0.50} \mathrm{In}_{0.50} \mathrm{P}$ and InP measured at the In $K$ edge. In both cases the peak at $R \sim 2 \AA$ in the FT spectra, due to scattering from first NN P atoms, is similar for the binary and ternary
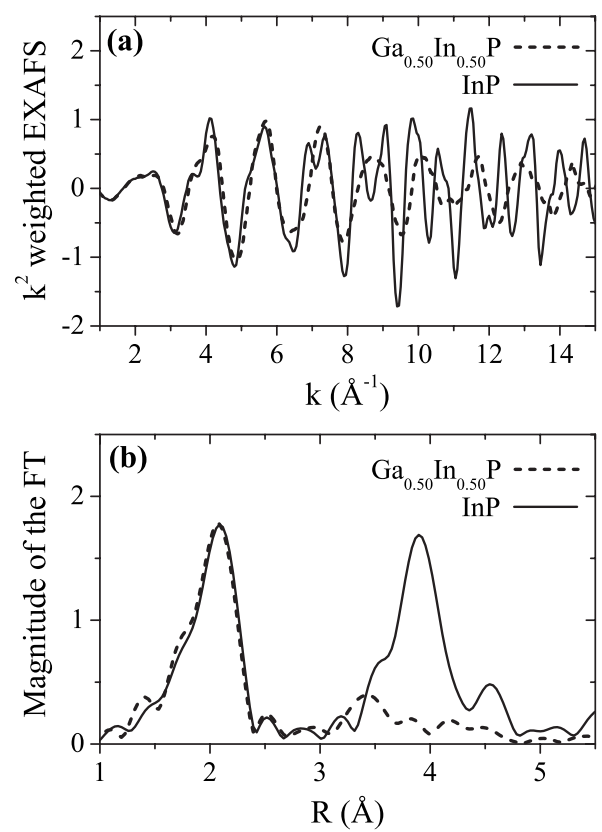

FIG. 2. (a) $k^{2}$-weighted EXAFS spectra of InP (solid line) and $\mathrm{Ga}_{0.50} \mathrm{In}_{0.50} \mathrm{P}$ (dashed line) recorded at the In $K$ edge versus the photoelectron wave number $k$. (b) Corresponding Fourier transforms as a function of the nonphase-corrected radial distance $R$ from the absorber.

alloys. The other two ternary compositions exhibited similar behavior (not shown). In contrast, the contributions from the second and third NN shells in the region $R \sim 3-5 \AA$ change significantly with composition. For the binaries, scattering occurs at second $\mathrm{NN}$ Ga or In and third NN P atoms; whereas for the ternaries, scattering occurs at second $\mathrm{NN} \mathrm{Ga}$ and $\mathrm{In}$ and third $\mathrm{NN} \mathrm{P}$ atoms.

FEFF8 (Ref. 23) was used to calculate phase shifts and scattering amplitudes $a b$ initio for $\mathrm{GaP}$, InP, and $\mathrm{Ga}_{1-x} \mathrm{In}_{x} \mathrm{P}$.

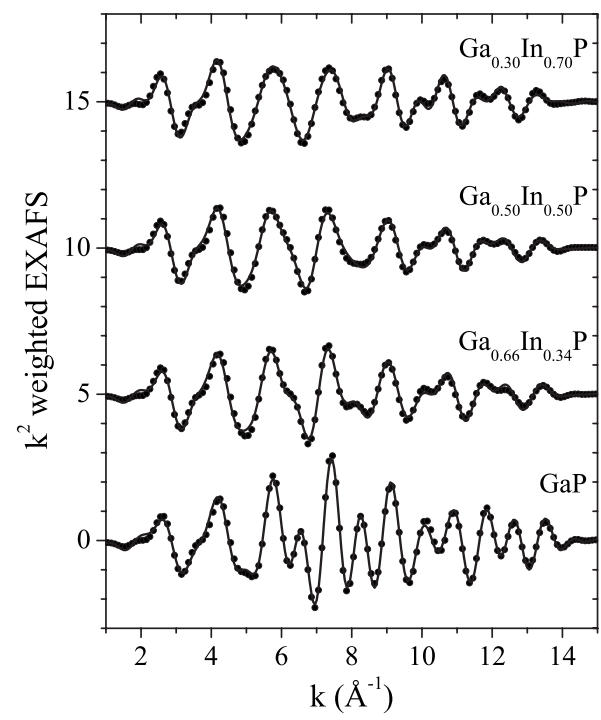

FIG. 3. $k^{2}$-weighted back-transformed experimental data (symbols) and best fits (lines) versus the photoelectron wave number $k$ for measurements taken at the Ga $K$ edge. For clarity only every second data point is displayed. 


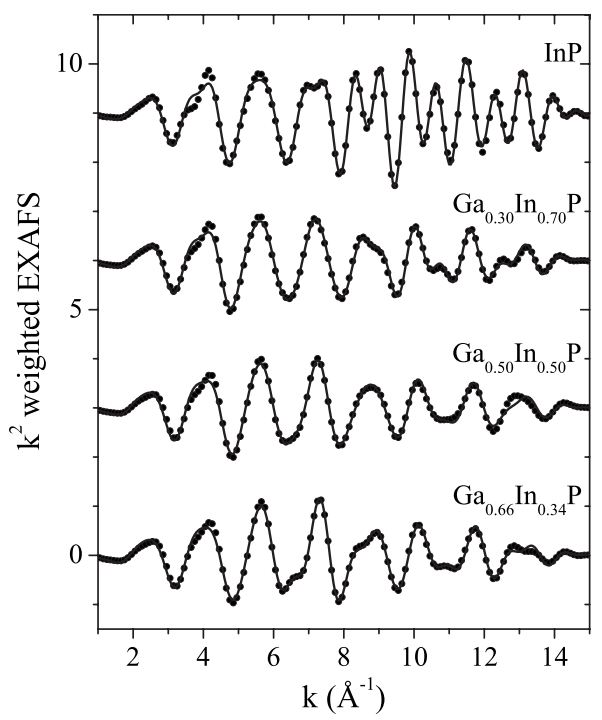

FIG. 4. $k^{2}$-weighted back-transformed experimental data (symbols) and best fits (lines) versus the photoelectron wave number $k$ for measurements taken at the In $K$ edge. For clarity only every second data point is displayed.

Fitting of the first three shells was performed in radial space over a range $R=1.4-4.6 \AA$ and $R=1.5-4.9 \AA$ for the $\mathrm{Ga}$ and In $K$ edge, respectively, using multiple $k$ weights of 1, 2, and 3. The amplitude reduction factor $\left(S_{0}^{2}\right)$ and the threshold energy $\left(E_{0}\right)$ were determined from the binary standards and were fixed while fitting the ternary samples. The coordination numbers for the first and third NN shells were set to the zinc-blende values of four and twelve, respectively. For the second shell, the sum of $\mathrm{Ga}$ and In coordination numbers was fixed to the zinc-blende value of twelve while the ratio of the two was chosen according to the composition determined by RBS. The interatomic distance and Debye-Waller factor for each of the following scattering paths were floated: first NN P, second NN Ga, second NN In, and third NN P, giving eight free parameters for each sample at each absorption edge measured. Note that fits performed with the second $\mathrm{NN} \mathrm{Ga}$ and second NN In distances set equal in accordance with the VCA model (not shown) were clearly inferior to those achieved with a bimodal distance distribution. For the binary compounds only the corresponding second NN Ga or In scattering path was considered. The resulting best fits (lines) are shown in Figs. 3 and 4 for measurement at the Ga and In $K$ edge, respectively, together with the backtransformed experimental data (symbols). The high quality of the fits is readily apparent.
The fits described above were achieved with a singlescattering approximation. Although multiple-scattering contributions are absent for a first-shell analysis of the zincblende structure, their potential influence must be considered for the analysis of higher shells such as that presented herein. We found that the inclusion of multiple-scattering contributions did not change the conclusions we present below. We attribute the negligible influence of multiple-scattering contributions to the presence of bond-length and bond-angle distortions in the ternary alloys under study. Multiple scattering is sensitive to the local geometric arrangement and thus to structural disorder. In our ternary alloys, the multiplescattering amplitudes were attenuated to a greater extent than single-scattering amplitudes and to a greater extent than in binary compounds. Furthermore, the FEFF calculation does not take into account the composition-dependent bond-length and bond-angle changes, reducing the accuracy for the phase shift and amplitude of the multiple-scattering paths. As described above, our model contains three single-scattering contributions (with two free parameters each) for the second and third shells. Increasing the number of paths by including multiple-scattering contributions did not improve the quality of the fits appreciably. ${ }^{24}$

\section{EXPERIMENTAL RESULTS}

\section{A. Interatomic distance distributions}

The structural parameters for the first three shells are summarized in Tables I and II for measurements at the Ga and In $K$ edge, respectively. The interatomic distance and Debye-Waller factor for each of the four scattering contributions are listed. Figure 5 shows these first two moments of the distance distribution as a function of composition $x$. The mean value (the interatomic distance) is given in the left panel, the standard deviation (the Debye-Waller factor) is given in the right panel, and the different rows represent scattering from the different shells.

The first NN distance distribution is clearly bimodal [Fig. $5(\mathrm{a})]$. The values for Ga-P and In-P mean distances exhibit a linear dependence on the composition $x$ and are much closer to the corresponding binary values than to the VCA (dotted line). The slope is the same for both and equal to $\sim 20 \%$ of that of the VCA. The Debye-Waller factors are constant within experimental uncertainty [Fig. 5(b)]. Considering the first NN shell, the ternary thus resembles a mixture of the binary atomic environments, having two distance distributions corresponding to Ga-P and In-P bonds, with mean values and widths similar to those of the binary compounds.

TABLE I. Structural parameters for $\mathrm{GaP}$ and $\mathrm{Ga}_{1-x} \mathrm{In}_{x} \mathrm{P}$ samples measured at the $\mathrm{Ga} K$ edge.

\begin{tabular}{|c|c|c|c|c|c|c|c|c|}
\hline & \multicolumn{3}{|c|}{ Interatomic distance $[\AA]$} & \multicolumn{5}{|c|}{ Debye-Waller factor $\left[10^{-3} \AA^{2}\right]$} \\
\hline & $1^{\mathrm{st}} \mathrm{NN} \mathrm{P}$ & $2^{\text {nd }} \mathrm{NN} \mathrm{Ga}$ & $2^{\text {nd }} \mathrm{NN}$ In & $3^{\text {rd }} \mathrm{NN} \mathrm{P}$ & $1^{\mathrm{st}} \mathrm{NN} \mathrm{P}$ & $2^{\text {nd }} \mathrm{NN} \mathrm{Ga}$ & $2^{\text {nd }} \mathrm{NN}$ In & $3^{\text {rd }} \mathrm{NN} \mathrm{P}$ \\
\hline $\mathrm{GaP}$ & $2.342 \pm 0.006$ & $3.854 \pm 0.005$ & & $4.49 \pm 0.01$ & $2.7 \pm 0.8$ & $3.8 \pm 0.5$ & & $4 \pm 2$ \\
\hline $\mathrm{In}_{0.34} \mathrm{Ga}_{0.66} \mathrm{P}$ & $2.355 \pm 0.004$ & $3.93 \pm 0.02$ & $3.98 \pm 0.03$ & $4.60 \pm 0.02$ & $2.7 \pm 0.6$ & $7 \pm 2$ & $7 \pm 3$ & $11 \pm 4$ \\
\hline $\mathrm{In}_{0.50} \mathrm{Ga}_{0.50} \mathrm{P}$ & $2.361 \pm 0.004$ & $3.96 \pm 0.02$ & $4.01 \pm 0.02$ & $4.65 \pm 0.03$ & $3.0 \pm 0.6$ & $8 \pm 3$ & $7 \pm 2$ & $12 \pm 4$ \\
\hline $\mathrm{In}_{0.70} \mathrm{Ga}_{0.30} \mathrm{P}$ & $2.363 \pm 0.005$ & $4.00 \pm 0.04$ & $4.06 \pm 0.02$ & $4.72 \pm 0.03$ & $2.8 \pm 0.7$ & $7 \pm 5$ & $7 \pm 2$ & $11 \pm 4$ \\
\hline
\end{tabular}


TABLE II. Structural parameters for InP and $\mathrm{Ga}_{1-x} \mathrm{In}_{x} \mathrm{P}$ samples measured at the In $K$ edge.

\begin{tabular}{|c|c|c|c|c|c|c|c|c|}
\hline & \multicolumn{3}{|c|}{ Interatomic distance $[\AA]$} & \multicolumn{5}{|c|}{ Debye-Waller factor $\left[10^{-3} \AA^{2}\right]$} \\
\hline & $1^{\mathrm{st}} \mathrm{NN} \mathrm{P}$ & $2^{\text {nd }} \mathrm{NN} \mathrm{Ga}$ & $2^{\text {nd }} \mathrm{NN}$ In & $3^{\text {rd }} \mathrm{NN} \mathrm{P}$ & $1^{\text {st }} \mathrm{NN} \mathrm{P}$ & $2^{\text {nd }} \mathrm{NN} \mathrm{Ga}$ & $2^{\text {nd }} \mathrm{NN}$ In & $3^{\text {rd }} \mathrm{NN} \mathrm{P}$ \\
\hline $\mathrm{In}_{0.34} \mathrm{Ga}_{0.66} \mathrm{P}$ & $2.506 \pm 0.003$ & $3.98 \pm 0.01$ & $4.00 \pm 0.02$ & $4.62 \pm 0.02$ & $2.4 \pm 0.3$ & $5 \pm 1$ & $4 \pm 1$ & $11 \pm 3$ \\
\hline $\mathrm{In}_{0.50} \mathrm{Ga}_{0.50} \mathrm{P}$ & $2.512 \pm 0.003$ & $4.01 \pm 0.02$ & $4.05 \pm 0.02$ & $4.66 \pm 0.03$ & $2.4 \pm 0.4$ & $8 \pm 3$ & $8 \pm 2$ & $13 \pm 4$ \\
\hline $\mathrm{In}_{0.70} \mathrm{Ga}_{0.30} \mathrm{P}$ & $2.521 \pm 0.003$ & $4.07 \pm 0.03$ & $4.10 \pm 0.01$ & $4.74 \pm 0.02$ & $2.5 \pm 0.4$ & $7 \pm 4$ & $7 \pm 1$ & $11 \pm 3$ \\
\hline InP & $2.531 \pm 0.005$ & & $4.157 \pm 0.004$ & $4.85 \pm 0.01$ & $2.5 \pm 0.7$ & & $3.8 \pm 0.4$ & $5 \pm 2$ \\
\hline
\end{tabular}

The second NN distance distribution also features different mean values for the different atomic pairs. As apparent in Fig. 5(c), the Ga-Ga distance is smaller than the Ga-In distance which, in turn, is smaller than the In-In distance. Note that the values for the mixed pair determined at either the $\mathrm{Ga}$ or In $K$ edge agree very well. The interatomic distances of all three pairs follow a linear dependence in $x$ and have a similar slope. There is no obvious trend in the Debye-Waller factors when comparing the different atomic pairs [Fig. 5(d)] but there is a significant increase in the ternaries compared to the binaries. The second NN distance distributions thus still exhibit discrete mean distances corresponding to the different cation-cation pairs but the values are now much closer to the VCA.

The mean third NN distance of the Ga-P pair is still systematically lower than that of the In-P pair but the difference is now within experimental uncertainty [Fig. 5(e)]. The values show a linear dependence on $x$ and agree well with the VCA. The Debye-Waller factors for both atomic pairs are nearly identical for the ternaries and show a strong increase compared to GaP and InP [Fig. 5(f)]. The VCA thus provides a very good approximation for the interatomic distance of the third NN shell although the width of the distribution is significantly increased in the ternary alloys.

\section{B. Tetrahedral bond angles}

Using the first and second $\mathrm{NN}$ distances, the tetrahedral bond angles can be calculated. Table III and Fig. 6 show the values of $\Varangle$ Ga-P-Ga, $\Varangle$ Ga-P-In, and $\Varangle$ In-P-In for the two binaries and the three ternaries. The two binary values agree very well although they are slightly higher than the ideal zinc-blende value of $109.5^{\circ}$. This slight difference could be attributed to offsets in the absolute interatomic distance determination during the fitting, but it is clearly small compared to the change due to composition in the ternary alloy. As discussed in Sec. III A, the second NN distances follow the VCA more closely than the first NN distances. With increasing $x$ the Ga-Ga distance thus increases to a greater relative extent than the Ga-P distance, leading to a larger $\Varangle \mathrm{Ga}-\mathrm{P}-\mathrm{Ga}$ tetrahedral bond angle compared to the ideal zinc-blende value. Similarly, $\Varangle$ In-P-In is expected to decrease from the ideal value with decreasing $x$ and such behavior is readily apparent in Fig. 6. Furthermore, the values for all three angles show a linear dependence on $x$ with the same slope. The change in the tetrahedral bond angle with composition demonstrates that lattice mismatch is accommodated in the ternary alloy by adjusting both bond lengths and bond angles.

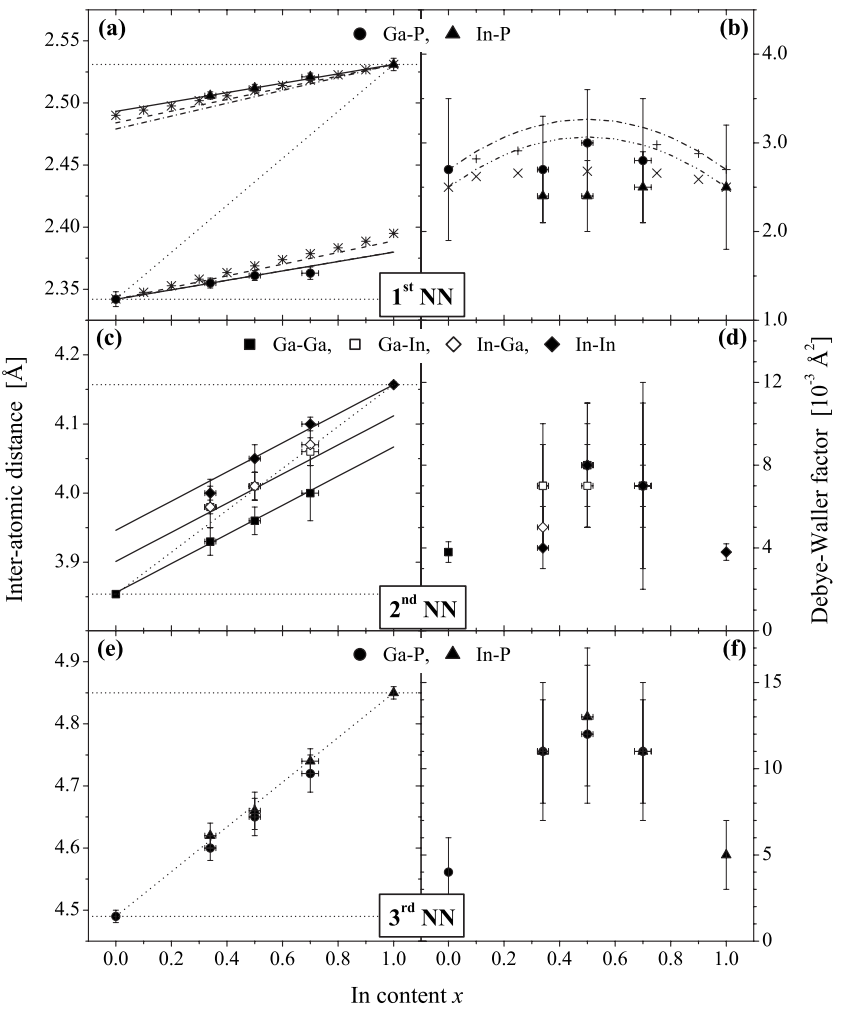

FIG. 5. Structural parameters determined for GaP, InP, and $\mathrm{Ga}_{1-x} \operatorname{In}_{x} \mathrm{P}$ as a function of composition $x$. Shown are the interatomic distances and Debye-Waller factors for scattering at the first NN P [(a) and (b)], at the second NN Ga or In [(c) and (d)] and at the third NN P [(e) and (f)] atoms. Experimental results: In panels (a), (b), (e), and (f) the values for the Ga-P and In-P pairs are denoted by full circles and triangles, respectively. In panels (c) and (d), the values for the Ga-Ga and In-In pairs are depicted as full squares and diamonds, respectively. Additionally, the values for the mixed cation-cation pair are shown as open squares and diamonds for measurement at the $\mathrm{Ga}$ and In $K$ edge, respectively. The dotted lines in panels (a), (c), and (e) represent the binary values and the corresponding VCA. Theoretical calculations: The solid lines in panel (a) represent the best fit yielding $\epsilon=0.80 \pm 0.04$. The other data sets show the calculations by Shih et al. (Ref. 11) (dashed line), Silverman et al. (Ref. 15) (dash-dotted line), and Balzarotti et al. (Ref. 8) (stars). The solid lines in panel (c) represent the calculations according to Cai and Thorpe (Ref. 29) using $\epsilon=0.8$ determined from panel (a). Panel (b) gives the calculations by Silverman et al. (crosses) and by Cai and Thorpe again with $\epsilon=0.8$ (dashdotted lines). For both calculations, the Ga-P value is higher than the In-P value for each composition $x$. For discussion of the various calculations see Sec. IV. 
TABLE III. Tetrahedral bond angles with $\mathrm{P}$ as central atom for $\mathrm{GaP}$, InP, and $\mathrm{Ga}_{1-x} \mathrm{In}_{x} \mathrm{P}$.

\begin{tabular}{lccc}
\hline [Degree $]$ & $\Varangle$ Ga-P-Ga & $\Varangle$ Ga-P-In & $\Varangle$ In-P-In \\
\hline $\mathrm{GaP}$ & $110.7 \pm 0.6$ & & \\
$\mathrm{In}_{0.34} \mathrm{Ga}_{0.66} \mathrm{P}$ & $113.1 \pm 1.2$ & $109.9 \pm 0.7$ & $105.9 \pm 0.9$ \\
$\mathrm{In}_{0.50} \mathrm{Ga}_{0.50} \mathrm{P}$ & $114.0 \pm 1.2$ & $110.7 \pm 1.1$ & $107.4 \pm 1.0$ \\
$\mathrm{In}_{0.70} \mathrm{Ga}_{0.30} \mathrm{P}$ & $115.6 \pm 2.2$ & $112.4 \pm 1.1$ & $108.8 \pm 0.6$ \\
$\mathrm{InP}$ & & & $110.4 \pm 0.5$ \\
\hline \hline
\end{tabular}

\section{THEORETICAL CALCULATIONS}

\section{A. Models for the first NN distance}

A number of models have been proposed to describe the atomic-scale structure of ternary alloys, most stemming from the 1980s. ${ }^{8,11-14}$ These early works sought to predict the first $\mathrm{NN}$ distance between atoms $\mathrm{B}$ and $\mathrm{C} d_{\mathrm{BC}}$ in the dilute limit of an impurity atom $\mathrm{B}$ in a crystal $\mathrm{AC} d_{\mathrm{BC}}^{\mathrm{AC}} \mathrm{B}$. The dimensionless relaxation parameter $\epsilon$ is defined as the difference between $d_{\mathrm{BC}}^{\mathrm{AC}: \mathrm{B}}$ and the (unperturbed) first NN distance of the host $d_{A C}^{0}$ relative to the difference in first NN distances of the two binaries $\epsilon=\left(d_{\mathrm{BC}}^{\mathrm{AC}: \mathrm{B}}-d_{\mathrm{AC}}^{0}\right) /\left(d_{\mathrm{BC}}^{0}-d_{\mathrm{AC}}^{0}\right)$. The VCA thus corresponds to $\epsilon=0$ (no relaxation) and the Pauling limit to $\epsilon$ $=1$ (full relaxation). The values of $\epsilon$ obtained with the different models described below are summarized in Table IV. The advantage of considering the dilute limit is the resulting symmetry around the impurity atom, which simplifies the calculation. Some authors then proposed a linear dependence for the first NN distance between the two end points as suggested by early experimental works. ${ }^{5}$

The simplest model is that of Shih et al. ${ }^{11}$ It considers an impurity atom B and calculates the displacement of the surrounding first $\mathrm{NN} \mathrm{C}$ atoms, keeping all other atoms fixed. A harmonic potential is assumed for the first NN interactions with a geometric approximation to correlate the B-C and A-C distances. A single force constant is used for both bond types. $^{26}$ The result is independent of the nature of the A, B, and $\mathrm{C}$ atoms and dependent only on the crystal geometry. For the zinc-blende structure, $\epsilon=0.75$ is obtained [see Table IV

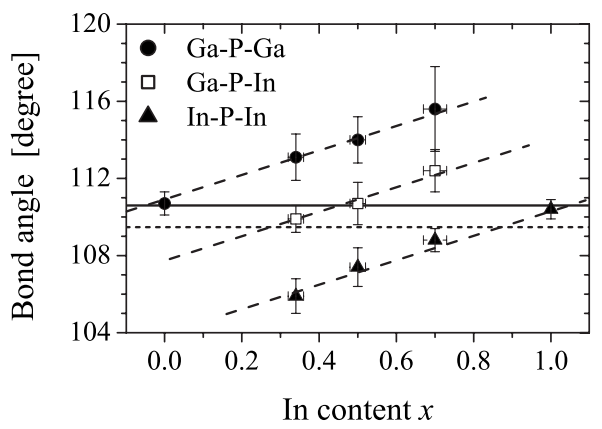

FIG. 6. Tetrahedral bond angles $\Varangle \mathrm{Ga}-\mathrm{P}$-Ga (full circles), $\Varangle$ Ga-P-In (open suares), and $\Varangle$ In-P-In (full triangles) as a function of composition $x$. The parallel dashed lines are a guide to the eye. The experimental value for the binaries and the ideal zinc-blende value are represented by a solid and a short-dashed lines, respectively.
TABLE IV. Values of $\epsilon$ as calculated by the different models discussed in Sec. IV. GaP:In refers to the case of In impurities in $\mathrm{GaP}$ while InP:Ga denotes Ga impurities in InP. The last two lines represent values determined experimentally.

\begin{tabular}{lcll}
\hline \hline Model & Ref. & GaP:In & InP:Ga \\
\hline Shih et al. & 11 & 0.75 & 0.75 \\
Martins and Zunger I & 12 & 0.63 & 0.73 \\
Martins and Zunger II & 12 & 0.73 & 0.77 \\
Chen and Sher & 13 & 0.70 & 0.80 \\
Balzarotti et al. & 8 & 0.78 & 0.72 \\
Srivastava et al. & 14 & 0.82 & 0.78 \\
Cai and Thorpe I & 29 & 0.72 & 0.72 \\
Cai and Thorpe II & 29 & 0.66 & 0.77 \\
Silverman et al. & 15 & 0.72 & 0.80 \\
Boyce and Mikkelsen & 6 & $0.80 \pm 0.05$ & $0.76 \pm 0.05$ \\
This work & & $0.80 \pm 0.04$ & $0.80 \pm 0.04$ \\
\hline \hline
\end{tabular}

and Fig. 5(a)]. Martins and Zunger ${ }^{12}$ use the valence force field (VFF) potential of Keating ${ }^{26,27}$ including bondstretching and bond-bending terms. Different force constants are applied for B-C and A-C bonds and the first and second NN shells around the impurity atom are relaxed (Martins and Zunger I). Including the bond-bending terms and relaxing the second NN shell change $\epsilon$ in opposite directions. Not including the two effects (Martins and Zunger II) thus gives a better result compared to experimental values than including only one effect. Chen and Sher ${ }^{13}$ consider not only the distortion energy due to bond stretching and bond bending but also "chemical" effects such as differences in binding energy, chemically driven charge redistribution, etc.

The work of Balzarotti et al. ${ }^{8}$ calculates the A-C and B-C distances for the entire compositional range, using the Keating VFF potential but without the bond-bending terms and fixing the second $\mathrm{NN}$ atoms. In the dilute limit it therefore corresponds to Martin and Zunger II except that two approximations [(i) including only the harmonic terms of the potential and (ii) approximating the geometric relation between A-C and B-C distances] used by all the models described above are not made by Balzarotti et al. Following the procedure proposed by Balzarotti et al., we have calculated the Ga-P and In-P distances and the corresponding $\epsilon$ [see Table IV and Fig. 5(a)]. Srivastava et al. ${ }^{14}$ take a somewhat different approach using first-principles atomic pseudopotentials but also calculate the first NN distances for the whole compositional range. The corresponding $\epsilon$ values are 0.78 and 0.82 for Ga-P and In-P, respectively.

\section{B. Calculations including the second NN shell}

All the models discussed so far concentrate on the first NN interatomic distance with a few simulations predicting multimodal distance distributions for the second NN shell. ${ }^{8}$ Based on the Kirkwood VFF potential, ${ }^{28}$ Cai and Thorpe ${ }^{29}$ derived expressions for the mean value and the width of the first NN distance distributions and for the second NN distances of the various atomic pairs. Their topological rigidity 
parameter $a^{* *}$ depends on the bond-stretching and bondbending force constants and is equivalent to $\epsilon$. The $\epsilon=a^{* *}$ values given in Table IV are derived from the force constants given by Cai and Thorpe assuming mean values (Cai and Thorpe I) or taking individual values (Cai and Thorpe II) for Ga-P and In-P bonds. An alternative approach is to determine the value that best represents the first NN distances-for our work $\epsilon=a^{* *}=0.80 \pm 0.04$ - and to use this value to predict other properties such as width of the first NN distance distribution and second NN mean distances [see Figs. 5(a)-5(c)].

The extensive work on $\mathrm{Ga}_{1-x} \operatorname{In}_{x} \mathrm{P}$ by Silverman et al. ${ }^{15}$ starts from a thermodynamic description of the alloy structure including energetic and entropic terms with configurational, positional, and vibrational contributions. Among other properties, the first and second NN distance distributions, as well as bond angle distributions, are derived [see Table IV and Figs. 5(a) and 5(b)]. In both works of Cai and Thorpe and Silverman et al., the standard deviation of the first NN distance distribution is calculated for a temperature of $0 \mathrm{~K}$, giving a value of zero for the two binaries. It can therefore be viewed as the purely static contribution to the Debye-Waller factor. Zero-point motion leads to a small but positive contribution to the Debye-Waller factor even at $0 \mathrm{~K}$. Our measurements were performed at $10-20 \mathrm{~K}$ and thus there will also be a thermal contribution. The resulting Debye-Waller factor for the binaries is small but nonzero. To compare experimental results with the calculations, the binary value (determined experimentally) has been added to all calculated values.

\section{DISCUSSION}

\section{A. First NN distance distribution}

The so-called $\mathbf{Z}$ plot, characteristic of the first NN distances in bulk ternary alloys, has been observed in many materials including $\mathrm{Ga}_{1-x} \operatorname{In}_{x}$ As (Ref. 5) and other III-V compounds, ${ }^{6,7}$ various II-VI ternary alloys, ${ }^{6-9}$ and in $\mathrm{K}_{1-x} \mathrm{Rb}_{x} \mathrm{Br}$ and $\mathrm{RbBr}_{1-x} \mathrm{I}_{x} \cdot{ }^{10,30}$ The experimental behavior of the III-V bulk ternary alloys is characterized by $\epsilon$ $=0.75-0.80$ and a linear dependence of the first NN distances on composition $x$. The situation is somewhat different in epitaxially grown thin films constrained by the substrate as shown by Woicik et al. ${ }^{31,32}$ for the case of buried $\mathrm{Ga}_{1-x} \mathrm{In}_{x} \mathrm{As}$ thin layers grown on InP. Here the first NN distances were observed to decrease with composition $x$ and also exhibited a slight nonlinear bowing of the curves. The authors conclude that the tetragonal distortion due to the external strain imposed by the substrate opposes the natural distance distortions due to alloying. Our work for bulk $\mathrm{Ga}_{1-x} \operatorname{In}_{x} \mathrm{P}$ yields $\epsilon$ $=0.80 \pm 0.04$ for both atomic pairs and a linear dependence on $x$. It therefore agrees very well with the results of other III-V bulk alloys including the sole previous report for $\mathrm{Ga}_{1-x} \operatorname{In}_{x} \mathrm{P}{ }^{6}$

Table IV shows that all reported models yield $\epsilon$ values between 0.63 and 0.82 and thus, on average, slightly underestimate the experimentally determined extent of relaxation. Excellent agreement is found with the calculations by Srivastava et al. Comparing the different models, the following conclusions can be drawn: (i) Using the different values of force constants reported in the literature ${ }^{13,26,29}$ for any given model yields only small differences in $\epsilon(\leq 0.02)$ that are much smaller than the variation due to the use of different models. (ii) The choice of approximations is the most crucial factor to the resulting $\epsilon$ value. As discussed by Martins and Zunger, including neither the bond-bending terms in the VFF potential nor the relaxation of the second NN shell gives better results than including only one of the two effects. Using a harmonic approximation to the VFF potential and/or approximating the geometric relationship between the A-C and $\mathrm{B}-\mathrm{C}$ distances (Martins and Zunger II versus Balzarotti et al., see Sec. IV A and Table IV) not only changes the absolute values of $\epsilon$ but also the ratio between the values for In impurities in $\mathrm{GaP}$ (GaP:In) and Ga impurities in InP (In$\mathrm{P}: \mathrm{Ga})$. As Cai and Thorpe argue, the relaxation in semiconductors extends out to a long range. Nevertheless, the simple models relaxing only the first NN shell (Shih et al., Martins and Zunger II, and Balzarotti et al.) yield $\epsilon$ values similar to those of the more complex calculations. It is therefore very difficult to judge the appropriateness of a certain approximation without performing the complete calculation and comparing the result with experiments such as those presented herein. (iii) Most models give different $\epsilon$ values for GaP:In and InP:Ga due to the different force constants of the two binaries. This is not observed experimentally. Similar to the case of bulk $\mathrm{Ga}_{1-x} \mathrm{In}_{x} \mathrm{As},{ }^{29}$ neither the bowing nor the $\epsilon$ values predicted by Cai and Thorpe when taking into account the different force constants (Cai and Thorpe II) are apparent in the experiments. The actual geometric arrangement determined by the energy balance of bond stretching versus bond bending appears insensitive to the difference in force constants. Instead, energy minimization favors a linear dependence of the lattice constant on composition $x$. In strained epitaxially grown thin films, on the other hand, the lattice constant is not free to adjust according to alloy composition and hence Vegard's law is not obeyed. Under such circumstances, the different force constants of the two binaries lead to a bowing of the curves as demonstrated by Woicik et $a l .{ }^{31,32}$ for the case of strained $\mathrm{Ga}_{1-x} \mathrm{In}_{x} \mathrm{As}$ thin films.

The experimentally determined Debye-Waller factor of the first NN shell is essentially the same for the binary and all ternary compositions [Fig. 5(b)], similar to the findings for other III-V and II-VI compounds. ${ }^{5-8}$ The values predicted by Silverman et al. are closer to the experimental results than those of Cai and Thorpe (assuming $\epsilon=a^{* *}=0.80$ ), although both are within experimental uncertainty. Both models predict an increased Debye-Waller factor for the ternaries compared to the binaries that is not observed experimentally.

\section{B. Second and third NN distance distributions}

For $\mathrm{Ga}_{1-x} \mathrm{In}_{x} \mathrm{As}$, Mikkelsen and Boyce $^{5}$ find that the As-As distance distribution is clearly bimodal (corresponding to a bridging $\mathrm{Ga}$ or In atom) and strongly deviates from the VCA. In contrast, the cation-cation mean distances are much closer to the VCA. Nevertheless, they still systematically follow the relation Ga-Ga $<\mathrm{Ga}-\mathrm{In}<\mathrm{In}-\mathrm{In}$, which is very similar to what is shown for $\mathrm{Ga}_{1-x} \operatorname{In}_{x} \mathrm{P}$ in this work. 
Experimental results for other materials are also consistent with a strong distortion of the sublattice occupied by a single atom species, whereas the mixed sublattice is close to the VCA albeit significantly broadened. ${ }^{7,9,10}$ As discussed in Sec. $\mathrm{V}$ A, most models give similar $\epsilon$ values despite their very different assumptions, but the model by Cai and Thorpe is the only one that derives expressions for the mean second $\mathrm{NN}$ distances. As for $\mathrm{Ga}_{1-x} \mathrm{In}_{x} \mathrm{As}$, the predictions agree very well with the measurements when $\epsilon=a^{* *}=0.80$, determined from a best fit of the first NN distances, is used. The calculations by Silverman et al. are also in good qualitative agreement with the experimental findings (not shown). Both the relation between the distances of the three different pairs, namely, Ga-Ga $<\mathrm{Ga}-\mathrm{In}<\mathrm{In}$-In as well as the broadening of the distributions from the binaries toward $x=0.5$ can be clearly seen [Figs. 5(c) and 5(d)]. So far, there are no predictions for the third NN shell distance distribution. It can be concluded from the present work, however, that the VCA is a good approximation for the mean distance of the third NN atoms, although the distribution is significantly broadened.

\section{Tetrahedral bond angles}

The distribution of tetrahedral bond angles with a central $\mathrm{P}$ atom shown by Silverman et al. does not differentiate between the various possible cation combinations. The mean value is relatively composition independent and agrees well with the ideal zinc-blende value. If we assume (as suggested by Fig. 6) that the linear slope is the same for all three types of angles and that $\Varangle \mathrm{Ga}-\mathrm{P}-\mathrm{Ga}(x=0)=\Varangle \mathrm{Ga}-\mathrm{P}-\mathrm{In}(x=0.5)$ $=\Varangle \operatorname{In}-\mathrm{P}-\operatorname{In}(x=1)=\alpha$, with $\alpha$ being the binary value, then the weighted average over all angles with a central $\mathrm{P}$ atom is indeed $\alpha$ and independent of composition $x$. Given the small change in mean first NN distances with composition, the fact that the higher NN shells follow Vegard's law much more closely can only be realized by adjusting the tetrahedral bond angles. Bond bending is thus energetically favored over bond stretching and the lattice mismatch is mostly accommodated by bond-angle relaxation rather than bond-length relaxation. A similar behavior is observed in epitaxially grown strained thin films of $\mathrm{Ga}_{1-x} \mathrm{In}_{x} \mathrm{As},{ }^{25} \operatorname{InAs}_{x} \mathrm{P}_{1-x},{ }^{33}$ and $\mathrm{Si}_{1-x} \mathrm{Ge}_{x} .{ }^{34}$ The first NN distances remain close to those of the binary compounds and the tetragonal strain is accommodated primarily by bond-angle distortions.

\section{CONCLUSION}

EXAFS was used to measure the local atomic environment around $\mathrm{Ga}$ and $\mathrm{In}$ atoms in $\mathrm{Ga}_{1-x} \mathrm{In}_{x} \mathrm{P}$ alloys as a func- tion of composition. The interatomic distance and DebyeWaller factor were determined for all atomic pairs in the first three NN shells. The first NN distance distribution is bimodal. The mean value for each contribution (Ga-P and In-P) is composition dependent although similar to the distances in the corresponding binaries. A relaxation parameter of $\epsilon$ $=0.80 \pm 0.04$ together with a linear dependence on $x$ describes well the interatomic distances of both pairs. Most models slightly underestimate $\epsilon$ but excellent agreement is found with the calculations by Srivastava et al. ${ }^{14}$ The second NN distance distribution exhibits three peaks corresponding to Ga-Ga, Ga-In and In-In. The mean distances are closer to the VCA than for the first NN shell but the widths are significantly increased compared to the two binaries. Using $\epsilon$ $=a^{* *}=0.80$, determined from the first NN distances, the expressions derived by Cai and Thorpe ${ }^{29}$ for the second NN distances agree well with the experimental values. $\mathrm{Ga}_{1-x} \operatorname{In}_{x} \mathrm{P}$ therefore behaves like $\mathrm{Ga}_{1-x} \mathrm{In}_{x}$ As for which the distances of all atomic pairs within the first and second NN shells are also well represented by the model of Cai and Thorpe when using the same value of $\epsilon=a^{* *}=0.80$. The results presented here also agree with the extensive calculations by Silverman et al. ${ }^{15}$ for $\mathrm{Ga}_{1-x} \mathrm{In}_{x} \mathrm{P}$ alloys. For the third $\mathrm{NN}$ shell, the mean Ga-P distance is still systematically smaller than that of In-P but both agree well with the VCA within experimental uncertainty. Thus, the averaging over various local atomic arrangements makes the VCA a valid approximation for the third NN shell, but the distribution is significantly broader compared to the binary compounds. The tetrahedral bond angle around a central $\mathrm{P}$ atom changes linearly with composition $x$ increasing $(\Varangle \mathrm{Ga}-\mathrm{P}-\mathrm{Ga})$ or decreasing ( $\Varangle \mathrm{In}-\mathrm{P}-\mathrm{In})$ by $\sim 6.5^{\circ}$ with respect to the binary value. This clearly demonstrates that the lattice mismatch is accommodated in the ternary structure by both bond-length and bond-angle relaxations although primarily via the latter.

\section{ACKNOWLEDGMENTS}

We thank H. H. Tan for the growth of the $\mathrm{Ga}_{1-x} \mathrm{In}_{x} \mathrm{P}$ epilayers. We also thank the staff at the Photon Factory for their continued work and effort. The authors were supported by the Australian Synchrotron Research Program. P.K. and M.C.R. further acknowledge the support of the Australian Research Council.

\footnotetext{
${ }^{1}$ Sadao Adachi, Physical Properties of III-V Semiconductor Compounds (Wiley, New York, 1992).

${ }^{2}$ L. Vegard, Z. Phys. 5, 17 (1921).

${ }^{3}$ L. Nordheim, Ann. Phys. 401, 607 (1931); 401, 641 (1931).

${ }^{4}$ L. Pauling and M. L. Huggins, Z. Kristallogr. 87, 205 (1934).

${ }^{5}$ J. C. Mikkelsen, Jr. and J. B. Boyce, Phys. Rev. Lett. 49, 1412 (1982); J. C. Mikkelsen, Jr. and J. B. Boyce, Phys. Rev. B 28 , 7130 (1983)
}

${ }^{6}$ J. B. Boyce and J. C. Mikkelsen, Jr., Ternary and Multinary Compounds (Materials Research Society, Pittsburgh, 1987), p. 359.

${ }^{7}$ Z. H. Wu, K. Q. Lu, Y. R. Wang, J. Dong, H. F. Li, C. X. Li, and Z. Z. Fang, Phys. Rev. B 48, 8694 (1993).

${ }^{8}$ A. Balzarotti, M. Czyżnyk, A. Kisiel, N. Motta, M. Podgórny, and M. Zimnal-Starnawska, Phys. Rev. B 30, 2295 (1984); A. Balzarotti, N. Motta, A. Kisiel, M. Zimnal-Starnawska, M. T. 
Czyżnyk, and M. Podgórny, ibid. 31, 7526 (1985).

${ }^{9}$ A. Balzarotti, Ternary and Multinary Compounds (Materials Research Society, Pittsburgh, 1987), p. 333.

${ }^{10}$ J. B. Boyce and J. C. Mikkelsen, Jr., Phys. Rev. B 31, 6903 (1985).

${ }^{11}$ C. K. Shih, W. E. Spicer, W. A. Harrison, and A. Sher, Phys. Rev. B 31, 1139 (1985).

${ }^{12}$ J. L. Martins and A. Zunger, Phys. Rev. B 30, 6217 (1984).

${ }^{13}$ A.-B. Chen and A. Sher, Phys. Rev. B 32, 3695 (1985).

${ }^{14}$ G. P. Srivastava, J. L. Martins, and A. Zunger, Phys. Rev. B 31, 2561 (1985).

${ }^{15}$ A. Silverman, A. Zunger, R. Kalish, and J. Adler, Phys. Rev. B 51, 10795 (1995).

${ }^{16}$ C. Ghezzi, R. Magnanini, A. Parisini, L. Tarricone, E. Gombia, and M. Longo, Phys. Rev. B 77, 125317 (2008).

${ }^{17}$ M. Muccini, Nat. Mater. 5, 605 (2006).

${ }^{18}$ D. J. Friedman and S. R. Kurtz, Prog. Photovoltaics 10, 331 (2002).

${ }^{19}$ C. S. Jiang, H. R. Moutinho, J. F. Geisz, D. J. Friedman, and M. M. Al-Jassim, Appl. Phys. Lett. 81, 2569 (2002).

${ }^{20}$ A. de la Zerda and S. S. Gambhir, Nat. Nanotechnol. 2, 745 (2007).

${ }^{21}$ M. Newville, J. Synchrotron Radiat. 8, 322 (2001).
${ }^{22}$ B. Ravel and M. Newville, J. Synchrotron Radiat. 12, 537 (2005).

${ }^{23}$ A. L. Ankudinov, B. Ravel, J. J. Rehr, and S. D. Conradson, Phys. Rev. B 58, 7565 (1998).

${ }^{24}$ We note, in contrast, that Tormen et al. (Ref. 25) did find it necessary to include multiple-scattering contributions in their study of thin, strained $\mathrm{Ga}_{1-x} \mathrm{In}_{x}$ As layers.

${ }^{25}$ M. Tormen, D. DeSalvador, A. V. Drigo, F. Romanato, F. Boscherini, and S. Mobilio, Phys. Rev. B 63, 115326 (2001).

${ }^{26}$ R. M. Martin, Phys. Rev. B 1, 4005 (1970).

${ }^{27}$ P. N. Keating, Phys. Rev. 145, 637 (1966).

${ }^{28}$ J. G. Kirkwood, J. Chem. Phys. 7, 506 (1939).

${ }^{29}$ Y. Cai and M. F. Thorpe, Phys. Rev. B 46, 15872 (1992); Y. Cai and M. F. Thorpe, ibid. 46, 15879 (1992).

${ }^{30}$ A. DiCicco, E. Principi, and A. Filipponi, Phys. Rev. B 65, 212106 (2002)

${ }^{31}$ J. C. Woicik, Phys. Rev. B 57, 6266 (1998).

${ }^{32}$ J. C. Woicik, J. A. Gupta, S. P. Watkins, and E. D. Crozier, Appl. Phys. Lett. 73, 1269 (1998).

${ }^{33}$ S. Pascarelli, F. Boscherini, C. Lamberti, and S. Mobilio, Phys. Rev. B 56, 1936 (1997).

${ }^{34}$ J. C. Woicik, C. E. Bouldin, K. E. Miyano, and C. A. King, Phys. Rev. B 55, 15386 (1997). 